Introducing polar co-ordinates, we obtain the total rotation $\Delta \psi$ with respect to the origin of coordinates as follows:

$$
\Delta \psi=\Delta \mu+\Delta \varphi=\frac{\frac{\rho}{R} \cos u}{1+\frac{\rho}{R} \cos u}+\frac{\frac{\rho}{R} \sin u}{1+\frac{\rho}{R} \cos u}
$$

The construction of Laue patterns is reduced to the construction of reflection projections of the irradiated volume on the plane normal to the direction of the X-ray beam. In the construction of these projections, it is necessary to take into account the fact that the projecting (reflected) rays are not parallel. In Figs. $2 a$ and $2 b$ are shown the theoretical (calculated only for four zones) and the experimental Laue patterns from bent $(R=26 \mathrm{~mm}$.) sodium chloride crystals $(0.8 \mathrm{~mm}$. thick). The Laue patterns are obtained with a flat thin beam $(3 \mathrm{~mm}$. $\times 0.2 \mathrm{~mm}$.) of $\mathrm{X}$-rays parallel to the bending axis in the plane of axial section, to eliminate the ordinary rotation. In Figs. $3 a$ and $3 b$ are shown Laue patterns from the same crystal (but $1.7 \mathrm{~mm}$. thick). These patterns are obtained with a thin cylindrical beam of X-rays directed along the radius of bending.

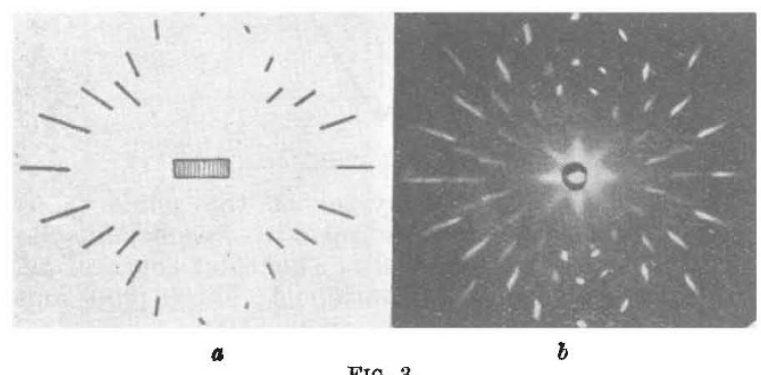

It is evident that experimental and theoretical Laue patterns are identical. The very small difference in the length of the theoretical and experimental Laue spots is explained by the divergence of the beam and the transverse deformation.

The coincidence of the theoretical and experimental Laue patterns shows that the crystal in the process of plastic deformation behaves as a mosaic aggregate of blocks, which change their orientation nearly independently ${ }^{2}$.

Physical-Technical Institute of the Ural,

A. P. Komar.

Sosnovka 2, Leningrad.

1 Schmid, E. und Boas, W., "Kristallplastizität", p. 62, Berlin (1935). ' Joffe, A., Kirpitschewa, M. W. und Lewitzky,'M. A., Z.Phys., 22, 286 (1924).

\section{Excitation of $\gamma$-Rays by Fast Neutrons}

IN the course of experiments on the excitation of $\gamma$-rays by slow neutrons already reported, wo have noticed that in some cases an appreciable amount of observed $\gamma$-rays was due to fast neutrons. Recently, we have investigated the emission of $\gamma$-rays by the bombardment of fast neutrons, and have obtained an interesting result as to the correlation of the crosssection for the exciting $\gamma$-rays with the atomic number. The experimental arrangements were essentially the same as those already described. The substance to be examined was surrounded by $\mathrm{B}_{2} \mathrm{O}_{3}$, and we believe that most of the neutrons reaching the substance retained their initial energy, which was about
$2 \times 10^{6}$ e.v., in our case of D-D neutrons. The result is tabulated in an accompanying table. The figures given in the second column are the relative cross-section per atom in an arbitrary scale. The $\gamma$-ray activity did not remain after the bombardment was ceased.

TABLE 1.

\begin{tabular}{|ll|l|ll|r|}
\hline 3 & $\mathrm{Li}$ & $-0.04 \pm 0.4$ & 26 & $\mathrm{Fe}$ & $2 \cdot 2 \pm 0.2$ \\
6 & $\mathrm{C}$ & $-0.1 \pm 0.3$ & 27 & $\mathrm{Co}$ & $2.1 \pm 0.34$ \\
7 & $\mathrm{~N}$ & $-0.05 \pm 0.3$ & 28 & $\mathrm{Ni}$ & $1.3 \pm 0.2$ \\
8 & $\mathrm{O}$ & $-0.01 \pm 0.1$ & 29 & $\mathrm{Cu}$ & $2 \cdot 6 \pm 0.2$ \\
9 & $\mathrm{~F}$ & $0.68 \pm 0.15$ & 30 & $\mathrm{Zn}$ & $2 \cdot 6 \pm 0.4$ \\
11 & $\mathrm{Na}$ & $0.60 \pm 0.23$ & 35 & $\mathrm{Br}$ & $2.5 \pm 0.3$ \\
12 & $\mathrm{Mg}$ & $0.05 \pm 0.23$ & 47 & $\mathrm{Ag}$ & $5.5 \pm 0.3$ \\
13 & $\mathrm{Al}$ & $0.42 \pm 0.1$ & 48 & $\mathrm{Cd}$ & $11.8 \pm 1.0$ \\
15 & $\mathrm{P}$ & $0.43 \pm 0.1$ & 50 & $\mathrm{Sn}$ & $5.1 \pm 1.1$ \\
16 & $\mathrm{~S}$ & $0.39 \pm 0.1$ & 51 & $\mathrm{Sb}$ & $8.1 \pm 1.0$ \\
17 & $\mathrm{Cl}$ & $0.05 \pm 0.2$ & 53 & $\mathrm{I}$ & $6.1 \pm 1.2$ \\
19 & $\mathrm{~K}$ & $0.05 \pm 0.3$ & 56 & $\mathrm{Ba}$ & $-0.6 \pm 1.5$ \\
20 & $\mathrm{Ca}$ & $-0.16 \pm 0.20$ & 79 & $\mathrm{Au}$ & $6.1 \pm 1.2$ \\
22 & $\mathrm{Ti}$ & $1.2 \pm 0.3$ & 80 & $\mathrm{Hg}$ & $5.4 \pm 2.4$ \\
24 & $\mathrm{Cr}$ & $3.0 \pm 1.1$ & 82 & $\mathrm{~Pb}$ & $0.2 \pm 0.3$ \\
25 & $\mathrm{Mn}$ & $1.7 \pm 0.4$ & 83 & $\mathrm{Bi}$ & $1.0 \pm 1.1$ \\
\hline
\end{tabular}

We shall first consider the elements with atomic number $Z$ lower than 30. For $Z \leqslant 8$ the crosssection is so small that we could detect no $\gamma$-rays. At $Z=9$ the emission becomes suddenly noticeable, and the cross-section decreases then with the increasing atomic number up to $Z=16$ (with the exception of $\mathrm{Mg}$ ). At $Z=17$ it falls suddenly down to zero and remains so up to $Z=20$. An intense emission of $\gamma$-rays begins again at $Z=22$. (The element $Z=21$ was not investigated.)

Thus we notice discontinuities at $8-9,16-17$ and $20-22$; that at $16-17$ is not so prominent, and it needs further precise measurement before we can decide as to its reality. The position of these discontinuities coincides with the positions where the regularity in the scheme of the stable isotopes changes discontinuously. This coincidence may not be merely an accidental one, or it may be deeply connected with the internal structure of the atomic nuclei of light elements.

On account of the small number of elements investigated, we cannot draw further conclusions as to the elements of high atomic number, except to say that the cross-section does not seem to be a smooth function of the atomic number. The absolute value of the cross-section is roughly estimated to be of the order of $10^{-24} \mathrm{~cm} .^{2}$ for copper. Our results are not directly comparable with those of Lea on account of the difference in energy of the neutrons.
S. KIKUOH
H. Aokr.
K. Husimar.

Physical Institute,

Imperial University, Osaka.

Jan. 10.

\section{A Provisional Map of a Human Chromosome}

By studying the simultaneous segregation of several genes in multiple heterozygotes, chromosome maps have been constructed for Drosophila, Zea and a few other organisms, and it has been shown that the order of the genes so deduced corresponds to a physical reality. It seemed likely that such maps could only be constructed very slowly for man, since very few pedigrees record the segregation of more than one gene at a time. In spite of this, indications of linkage between pairs of autosomal genes have been secured by Penrose ${ }^{1}$ and Bell ${ }^{2}$. 